\title{
CORRELATION BETWEEN THE RESULT EFFICIENCY INDICATORS OF SUCCESS IN TABLE TENNIS
}

\author{
Joško Sindik ${ }^{1}$, Nicolae Ochiana ${ }^{2}$, and Miran Kondrič ${ }^{3}$ \\ ${ }^{1}$ Institute for Anthropological Research, Zagreb, Croatia \\ ${ }^{2}$ Faculty of Health, Sports and Human Movement Sciences, University "Vasile Alecsandri" \\ of Bacau, Romania \\ ${ }^{3}$ Faculty of Sport, University of Ljubljana, Slovenia
}

\section{SUMMARY}

Amongst different methods of quality analysis of the table tennis player's game, the basic idea of this study was to detect those indicators (data) for collection of which only the final result in particular competitions in a larger number of events, of one game, or certain sets in one table tennis game would be sufficient. The basic aim of the research is to establish to what extent the indexes and variables derived directly from the results of individual table tennis games could predict indexes which are described by the final result for an individual in a larger number of individual table tennis games. The research involves an analysis of an intentional sample of 956 table tennis players competing in various leagues during 2007. Results show that correlations between individual variables of the table tennis competitors' efficiency are statistically significant. The correlations between indexes of the competitor's efficiency are also statistically significant. In particular, we found positive (and significant) correlations between all the direct and indirect indicators of players' efficacy (medium strongly to strongly correlated). The correlation between individual variables and indexes of the table tennis competitor's efficiency, with the total efficacy index, are statistically significant. It can be concluded that all the indexes and variables can describe specific aspects of the performance in table tennis, predicting total efficacy of the players.

Key Words: analysis, competition, prediction, racquet sports.

\section{INTRODUCTION}

Besides in technical field, table tennis is also demanding in terms of tactical knowledge, technical skills, motor abilities and morphological characteristics, as well as appropriate conative characteristics necessary for a successful competition achievement (Grujić, 1975; Hudetz, 1982). Notational analysis is used by coaches and sport scientists to gather objective data on the performance of athletes. Systems for notational analysis are becoming increasingly sophisticated, reflecting the demands of coaches and scientists, as well as improvements in technology (Hughes \& Franks, 1997). In any sporting situation, especially table tennis, it is difficult, if not impossible, for coaches to notice and remember all the key events occurring within a training session or match, equipped only with their knowledge of the sport in question and their powers of observation. Yet, analysis based on accurate observation and recall is a key tool for improving future performance of table tennis players. In table tennis notational analysis is an objective way of recording the performance of the player, so that critical events in that performance can be quantified in a consistent and reliable manner. Table tennis is one of the fastest ball games in the world, it is therefore difficult for the coach to notice and remember all the key elements occurring within a game or training session. Franks and Miller (1991) have highlighted memory retention problems, with coaches able to recall only $30-50 \%$ of key performance factors they had witnessed, even with special training in observation. However, as in many other sports branches, in table tennis analysis based on accurate observation 
and recall is a prime tool for improving future performance of the player. Table tennis is one of the sports branches that still have little research or real analysis, compared to sports branches like tennis or badminton. When we consult scientific data, we care about details and how we can transfer it in to our own practices, or how we can use it in any way. The most accurate observation is simple analysing of result in table tennis game. We have chosen exactly such approach.

The basic question which is set by the professionals aiming to improve result effect of sportsmen or teams in different sports branches is - how to assess the game quality (Brčić, Viskić Štalec, \& Jaklinović Fressl, 1997). Amongst different manners of quality analysis of the table tennis player's game, the basic idea of research was to detect those indicators (data) for collection of which only the final result in particular competitions in larger number of events, in one table tennis match, certain sets in one table tennis match could be sufficient. Sindik (1999) performed that by implementation of variables which could directly be derived from the results of competitions, however, those variables one could reduce to a smaller number of indexes. In the world of table tennis, some studies can be found, but in general there is a lack of literature for this discipline (Baca, Baron, Leser, \& Kain, 2004; Đokić, 2008; Leser \& Baca, 2009; Sindik, 1999; Sindik \& Vidak, 2009; Wilson \& Barnes, 1998; Wu Xiao \& Escobar-Vargas, 2007). Due to these facts, this work seeks to establish written support for future studies on this sport based on the research carried out by the authors, the references cited, and information collected from racket sports in general.

The basic aim of the study is to establish to what extent indexes directly derived from the results of individual table tennis games could predict indexes that are described by the final result of an individual in a larger number of individual table tennis games. This kind of analysis could potentially be useful for the requirements of future planning for individual player training. The goals of the research are to determine: the correlation between individual variables of a table tennis competitors' efficiency (1); the correlation between indexes of efficiency, derived by summarizing several particular indexes (2); the correlation between individual variables of a table tennis competitors' efficiency, with the total efficacy index (3); the capacity for index and variable prognosis: game index, set index, games won, sets won, total efficacy index, on the basis of other indexes and variables of table tennis game efficiency and log variables (4).

In previous studies (Sindik, 1999; Sindik \& Juričević, 2007) we found that most of the intercorrela- tions between the indexes and intercorrelations between the variables of a table tennis player's efficacy were intermediately to medium strongly correlated. Statistically significant predictive value of the indicators used was determined for the prognosis of direct indicators of efficiency, on the basis of other indexes and variables. We can generally assume that all the indexes and the total efficacy index of a table tennis competitors' efficiency are statistically significantly correlated with a performance success. Consequently, indexes and variables of final competition success can be predicted by all the variables and indexes of competitors' efficiency.

\section{METHODS}

\section{Sample Entity}

A final sample of 956 table tennis players competing during 2007 in various recreational table tennis leagues in SOKAZ, which played in leagues, ranked from 1 to 20 , with a minimum of 33 to a maximum of 63 players in each league. In the final sample we selected the individuals who played a minimum number of 6 table tennis games. Each individual whose result was collected played at least 6 individual games in the observed period, while the maximum number of games that the individual could play during one competition was 66 . All the players were male, aged from 11 to 84 .

\section{Variables}

The variables in table tennis competition were defined and can be derived directly from the competition results. Dependent variables, i.e. predictors (in regression analysis) were direct efficacy indicators:

- Games won: total number of games won, for an individual.

- Sets won: total number of sets won, for an individual, from the total number of games played.

Independent variables were predictors derived directly from the results (indirect efficacy indicators):

\section{A. efficacy variables}

- Games lost. total number of games played in which an individual lost the game.

- Sets lost. total number of sets played in which an individual lost the set.

- Sets won with point difference: total numbers of sets won that were played on difference (won 11-9, 12-10 etc.).

- Sets lost with point difference: total number of sets won that were played on point difference (lost 9-11, 10-12, etc.). 
- Games won played in five sets. total number of games won that were played in five sets (result 3-2 for an individual).

- Games lost played in five sets: total number of games lost that were played in five sets (result 2-3 for an opponent).

- Games won after 0-2 in sets for opponent: number of games won in which an individual won after losing the first two sets (0-2 advantage of the opponent).

- Games lost after leading 2-0 in sets: number of games lost in which an individual lost after winning the first two sets (2-0 advantage of the player).

\section{B. log variables}

- Number of games played: total number of games played by an individual.

- Number of sets played: total number of sets played by an individual.

- Turnover games (lost after leading 2-0 and won after 0-2): total number of games won in which an individual won after losing the first two sets (0-2 advantage of the opponent) and the number of games lost in which an individual lost after winning the first two sets (0-2 advantage of the opponent).

- Games played in five sets: total number of games played in five sets (result 3-2 for an individual or 2-3 for the opponent);

- Sets played on point difference: total number of sets played on point difference (won 11-9, 12-10 etc. or lost 9-11, 10-12, etc.).

Indexes (indicators of efficiency of an individual) have been defined, which can be derived directly from competition results. These indexes are theoretically considered as a »composite of two particular variables, while their basic »logic « is a calculation of the ratio between the effectively accomplished number of cases and the maximum possible number of cases, in relation to the hypothetical indicators of efficiency in competitive situations.

As dependent indexes which are direct indicators of players' efficiency i.e. criteria (in regression analysis) have been determined (direct efficacy indicators):

- Game index: ratio between the number of games won and lost in all an individual's games: total number of games won is divided by the total number of games played;

- Set index: ratio between the number of sets won and lost, in all sets in games played by an individual. Total number of sets won is divided by the total number of sets played.

Independent indexes were predictors derived directly from the results (indirect efficacy indicators):
- Set played on point difference index: ratio between the number of sets won and lost in sets played on point difference (11-9, 12-10, 9-11, 10-12 etc.): the number of sets won on point difference is divided by the total number of sets played on point difference.

- Game played in five sets index: ratio between the number of won and lost games played in 5 sets (win 3-2 and loss 2-3); the number of games won in 5 sets is divided by the total number of games played in 5 sets.

- Turnover index: ratio between the number of games won and lost, in which an individual won after losing the first two sets (0-2 advantage of the opponent), and in games when an individual had an advantage to the opponent leading 2-0 (and finally lost the game).

Finally, the total efficacy index is the sum of these three indexes (set played on point difference, game played in five sets, turnover index), as a hypothetical measure of a player's total efficacy, and it is used as a criterion for three predictors (statistical variables): turnover games, games played in five sets, sets played on point difference.

\section{Procedure}

Data collection was performed by inspecting all the results of individuals (players-examinees) from the official web page of the Table Tennis Organization of Clubs and Activities of Zagreb (SOKAZ www.sokaz.hr). The total result for an individual in a larger number of individual table tennis games and sets was determined. All results were collected from two championships in a period during 2007 (spring and autumn seasons - championships), from different competition ranks in which the given team competed. The role of judges and audience was reduced to a minimum, while games were played for three sets won. As a rule, there are 12 teams in each SOKAZ league (league level), which played games in two-round championship system (each against each), one game at home, and the other as a guest (5 or 6 games as a guest/at home, per championship). On the level of the one team game, each individual plays 3 games against 3 opponents from the other team. So, at the level of the championship, the maximum number of games per one player would be 33 .

\section{Statistical Analysis}

All data analysis was performed using the SPSS 15.0 package. Descriptive statistics for all variables and indexes were calculated. Pearson correlations 
were calculated for determining the correlation between all the variables and indexes. Complete multiple regression analysis was used for calculating the prediction of the criteria variables: game index, set index, total efficacy index, games won and sets won.

\section{RESULTS}

Table 1 gives descriptive values for all variables and the efficiency indexes for table tennis players in championships in SOKAZ in 2007.
It turned out that most of the distributions of results for the variables and indexes are asymmetric (Table 1). The exceptions are the variables such as game index, set index (deviation with $p>.10$ ) and total efficacy index (deviation with $p>.20$ ), where distributions do not differ significantly from the Gauss distribution. Due to the different range of variables that are direct and indirect indicators of success (conditional frequency of occurrence of certain events such as the number of sets played on point difference),

\section{TABLE 1}

Descriptive statistics for all the variables and indexes of efficiency for players in the SOKAZ table tennis championships in 2007.

\begin{tabular}{|c|c|c|c|c|c|c|c|}
\hline 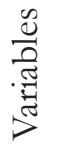 & MIN & MAX & 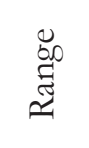 & $M$ & $S D$ & 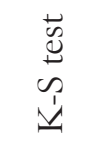 & $p$ \\
\hline 1. & 1.00 & 20.00 & 19 & 10.9377 & 5.8612 & 2.632 & .01 \\
\hline 2. & 1.00 & 64.00 & 63 & 25.1126 & 12.9873 & 2.087 & .01 \\
\hline 3. & .00 & 64.00 & 64 & 12.6639 & 7.5009 & 1.963 & .01 \\
\hline 4. & .00 & 35.00 & 35 & 12.5469 & 6.8782 & 2.002 & .01 \\
\hline 5. & 1.00 & 27.00 & 26 & 10.6708 & 5.7776 & 1.837 & .01 \\
\hline 6. & .00 & 19.00 & 19 & 5.3286 & 3.5917 & 2.839 & .01 \\
\hline 7. & .00 & 20.00 & 20 & 5.3270 & 3.3065 & 2.751 & .01 \\
\hline 8. & 1.00 & 8.00 & 7 & 2.2942 & 1.1544 & 7.348 & .01 \\
\hline 9. & .00 & 7.00 & 7 & 1.1475 & .9026 & 8.257 & .01 \\
\hline 10. & .00 & 6.00 & 6 & 1.1467 & .8747 & 8.829 & .01 \\
\hline 11. & 6.00 & 66.00 & 93 & 48.7866 & 19.6854 & 3.022 & .01 \\
\hline 12. & .00 & 66.00 & 91 & 25.337 & 16.3488 & 4.354 & .01 \\
\hline 13. & .00 & 62.00 & 62 & 23.4529 & 12.4673 & 2.057 & .01 \\
\hline 14. & 14.00 & 370.00 & 356 & 181.9425 & 75.6960 & 3.758 & .01 \\
\hline 15. & .00 & 283.00 & 283 & 93.7762 & 52.2356 & 1.842 & .01 \\
\hline 16. & .00 & 186.00 & 186 & 88.2239 & 40.4654 & 1.625 & .01 \\
\hline 17. & .00 & 1.00 & 1.00 & .4900 & .1764 & 1.056 & .10 \\
\hline 18. & .00 & 1.00 & 1.00 & .4868 & .2347 & 1.264 & .10 \\
\hline 19. & .00 & 1.00 & 1.00 & .4867 & .1575 & 5.900 & .01 \\
\hline 20. & .00 & 1.00 & 1.00 & .4836 & .2232 & 2.432 & .01 \\
\hline 21. & .00 & 1.00 & 1.00 & .4919 & .3349 & 6.310 & .01 \\
\hline 22. & .00 & 3.00 & 3.00 & 1.4623 & .5136 & 1.025 & .20 \\
\hline
\end{tabular}

Legend: 1. - League level; 2. - Sets played on point difference; 3. - Sets won with point difference; 4. - Sets lost with point difference; 5. - Games played in five sets; 6. - Games won played in five sets; 7. - Games lost played in five sets; 8. - Turnover games (lost after leading 2-0 and won after 0-2); 9. - Games won after 0-2 in sets for opponent; 10. - Games lost after leading 2-0 in sets; 11. - Number of games played; 12. - Games won; 13. - Games lost; 14. - Number of sets played; 15. - Sets won; 16. - Sets lost; 17. - Set index; 18. - Game index; 19. - Set play on point difference index; 20. - Game played in five sets index; 21. - Turnover index; 22. - total efficacy index; $M$ - Mean; MIN - Lowest value; MAX - Highest value; SD - Standard deviation; K-S test - Kolmogorov Smirnov test normality of the distribution; $\boldsymbol{p}$ - Probability. 
their direct comparison does not give us any information. However, for all indexes that have a total range from 0 to 1 , it was obtained the highest average score in the set index (among the direct indicators of success) and the turnover index (among the indirect indicators of success). On the other hand, the greatest variability was found for the turnover index, which is actually derived from the variables describing the rarest events in table tennis matches (turnover matches, matches won after loosing 0-2 in sets in favor of the opponent, lost matches after leading 2-0 in sets).

Table 2 shows that practically all correlations, apart from four are statistically significant (league level with: turnover index, set play on point difference index and total efficacy index, set play on point difference index with turnover index), and ranged from low (-.087 between league level and game play in five sets index) to very high (.729 between total efficacy index and turnover index). Along with game index (as potentially the most relevant index), the highest (positive and significant) values of correlation (together with the spurious correlation with set index) can be found for the following indexes: total efficacy index, then game played in five sets index, while the lowest correlation value was found for the set play on point difference index and turnover index, which is normally the least correlated with the remaining efficacy indexes. In lower competitive leagues, players on average have somewhat inferior direct and indirect indicators of table tennis efficacy. Statistically, the total efficacy index is significantly correlated (from average to high) with all efficacy indexes of a table tennis player.

\section{TABLE 2}

Pearson correlations between league rank and all the indexes of efficiency for the players in the SOKAZ table tennis championships in 2007.

\begin{tabular}{c|ccccccc}
\hline Variable & 1. & 2. & 3. & 4. & 5. & 6. & 7. \\
\hline 1. & 1.000 & $-.092^{*}$ & $-.096^{*}$ & $-.087^{*}$ & -.035 & -.001 & -.054 \\
\hline 2. & & 1.000 & $.981^{* *}$ & $.531^{* *}$ & $.232^{* *}$ & $.224^{* *}$ & $.468^{* *}$ \\
\hline 3. & & & 1.000 & $.457^{* *}$ & $.235^{* *}$ & $.183^{* *}$ & $.415^{* *}$ \\
\hline 4. & & & & 1.000 & $.149^{* *}$ & $.327^{* *}$ & $.678^{* *}$ \\
\hline 5. & & & & & 1.000 & .013 & $.576^{* *}$ \\
\hline 6. & & & & & & 1.000 & $.729^{* *}$ \\
\hline 7. & & & & & & & 1.000 \\
\hline
\end{tabular}

Legend: 1. - League level; 2. - Game index; 3. - Set index; 4. - Game played in five sets index;

5. - Set play on point difference index; 6. - Turnover index; 7. - Total efficacy index;

$*$ - correlation is significant at the $p<.05 ; * *$ - correlation is significant at the $p<.01$.

We have calculated the correlations between the league rank and all the variables of efficiency for the players in the SOKAZ table tennis championships in 2007 (we didn't show this correlation matrix because of its prolixity). It appears that practically all correlations are statistically significant and range from low $(r=.077 ; p<.05$ between games lost and sets won $)$ to very high $(r=.806 ; p<.01$; between sets played on point difference and games played in five sets), provided we exclude spurious correlations of variables, which are already invariably correlated to a certain extent. Along with the games won variable the highest (positive and significant) correlation values can be found for the following variables: sets won with difference, games won played in five sets, but also sets played, games played, sets played with difference, and games played in five sets. It is interesting that in this case the lowest (although significant and positive correlations) with the games won variable were found for turnover games, as well as for the variables games won after 0-2 in sets for opponent and games lost after leading 2-0 in sets. In lower competitive leagues, players on average have less frequent occurrences of efficacy indicators of table tennis players (direct and indirect), as well as log variables, which shows a negative indication of all (although low, but statistically significant) correlations between the league level and other variables. Statistically, the total efficacy index is significantly averagely correlated with all major variables, which constitute the efficacy indicators of table tennis players: games won, games won after $0-2$ in sets for opponent, sets won with difference, and games won played in five sets.

Table 3 shows that the game index, as the ratio of victories in table tennis games in relation to the total number of games, can be statistically predicted in a significant and successful manner, based on a group 
of three predictors, i.e. the remaining efficacy indexes: the set played on point difference index and the game played in five sets index (statistically significant predictors, with $p<.01)$.

\section{TABLE 3}

Prediction of the game index with the other game efficiency indexes for the players in two SOKAZ table tennis championships in 2007.

\begin{tabular}{llllcl}
\hline \multirow{2}{*}{ Regression } & $R$ & $R^{2}$ & $R_{c}^{2}$ & $F$ & $p$ \\
\cline { 2 - 6 } & $\mathbf{. 6 7 0}$ & $\mathbf{. 4 4 8}$ & $\mathbf{. 3 0 7}$ & $\mathbf{2 5 7 . 8 2}$ & $\mathbf{. 0 1}$ \\
\hline Variables & $\mathrm{B}$ & $S E$ & $\beta$ & $t$ & $p$ \\
\hline Game index (D) & -.036 & .021 & & -1.761 & .05 \\
\hline Set play on difference index $(\mathrm{P})$ & .588 & .037 & .394 & 15.866 & $\mathbf{. 0 1}$ \\
\hline Game played in five sets index $(\mathrm{P})$ & .458 & .028 & .436 & 16.405 & $\mathbf{. 0 1}$ \\
\hline Turnover index $(\mathrm{P})$ & .032 & .018 & .045 & 1.738 & .05 \\
\hline
\end{tabular}

Legend: $R$ - Multiple correlation coefficient; $R^{2}$ - Determinantion coefficient; $R_{c}^{2}$ - Corrected determination coefficient; $F$ - F-ratio; $p$ - Probability; B - Beta coefficient; $S E$ Standarda error of estimate; $\boldsymbol{B}$ - Beta standardize partial contribution; $\boldsymbol{t}$ - $\boldsymbol{t}$-test; D - Dependent; $\mathbf{P}$ - Predictor.

From Table 4 one can see that the set index, as the ratio of sets won in table tennis games in relation to the total number of sets played, can also be statistically predicted in a significant and successful manner, based on a group of three predictors the remaining efficacy indexes: the set played on point difference index and the game played in five sets index (statistically significant predictors, with $p<.01$ ).

\section{TABLE 4}

Prediction of the set index with the other game efficiency indexes for the players in two SOKAZ table tennis championships in 2007.

\begin{tabular}{llllcl}
\hline \multirow{2}{*}{ Regression } & $R$ & $R^{2}$ & $R_{c}^{2}$ & $F$ & $p$ \\
\cline { 2 - 6 } & $\mathbf{. 6 2 7}$ & $\mathbf{. 3 9 3}$ & $\mathbf{. 3 9 1}$ & $\mathbf{2 0 5 . 0 6}$ & $\mathbf{. 0 1}$ \\
\hline Variables & $\mathrm{B}$ & $S E$ & $\beta$ & $t$ & \multicolumn{1}{c}{$p$} \\
\hline Set index (D) & .114 & .016 & & 6.986 & $\mathbf{. 0 1}$ \\
\hline Set play on difference index $(\mathrm{P})$ & .480 & .029 & .429 & 16.430 & $\mathbf{. 0 1}$ \\
\hline Game played in five sets index $(\mathrm{P})$ & .278 & .022 & .352 & 12.627 & $\mathbf{. 0 1}$ \\
\hline Turnover index $(\mathrm{P})$ & .017 & .014 & .032 & 1.172 & .20 \\
\hline
\end{tabular}

Legend: $R$ - Multiple correlation coefficient; $R^{2}$ - Determinantion coefficient; $R_{c}^{2}$ - Corrected determination coefficient; $F$ - F-ratio; $p$ - Probability; B - Beta coefficient; $S E$ Standarda error of estimate; $\boldsymbol{B}$ - Beta standardize partial contribution; $\boldsymbol{t}$ - $\boldsymbol{t}$-test; D - Dependent; $\mathbf{P}$ - Predictor.

In both cases (Table 3 and 4), the turnover index was the statistically insignificant predictor.

It is clear (Table 5) that the games won variable, as the simplest but also the most relevant efficacy index in table tennis games, can be statistically predicted in a significant and successful manner, based on a group of three predictors, i.e. the remaining variables of a table tennis player's efficacy: sets won with point difference, games won played in five sets, and games lost played in five sets (all predictors are statistically significant with $p<.01)$.
Observing Table 6, one can see that sets won, as a similar efficacy index in table tennis games, can be statistically predicted in a significant and successful manner, based on a group of six predictors, i.e. the remaining variables of a table tennis player's efficacy: sets won with point difference and games won played in five sets (all predictors are statistically significant with $p<.01)$.

In both cases (Table 5 and 6 ), turnover variables were statistically insignificant predictors.

Table 7 shows that the game index can be statistically predicted in a significant and successful manner, 
based on a group of three predictors, i.e. neutral or log variables: sets played on point difference and sets played in five sets (statistically significant predictors, with $p<.01)$.

From Table 8, it can be deduced that the games won variable can be statistically predicted in a significant and successful manner, based on a group of three predictors, i.e. neutral or log variables, among which one is statistically significant predictor: sets played on point difference (with $p<.01$ ).

Finally, in Table 9 it is evident that the total efficacy variable, as a indicator of total result efficacy, can be statistically predicted in a significant and successful manner, based on a group of three predictors, i.e. neutral or log variables, among which all predictors are statistically insignificant.

\section{TABLE 5}

Prediction of the variable games won with game efficiency variables for the players in two SOKAZ table tennis championships in 2007.

\begin{tabular}{llllrl}
\hline \multirow{2}{*}{ Regression } & \multicolumn{1}{c}{$\mathrm{R}$} & $\mathrm{R}^{2}$ & \multicolumn{1}{c}{$\mathrm{R}_{c}^{2}$} & \multicolumn{1}{c}{$F$} & \multicolumn{1}{c}{$p$} \\
\cline { 2 - 6 } & $\mathbf{. 7 4 5}$ & $\mathbf{. 5 5 6}$ & $\mathbf{. 5 5 3}$ & $\mathbf{1 9 7 . 8 2}$ & $\mathbf{. 0 1}$ \\
\hline Variables & \multicolumn{1}{c}{$\mathrm{B}$} & \multicolumn{1}{l}{$S E$} & \multicolumn{1}{c}{$\beta$} & \multicolumn{1}{c}{$t$} & \multicolumn{1}{c}{$p$} \\
\hline Games won (D) & 6.069 & .935 & & 6.488 & $\mathbf{. 0 1}$ \\
\hline Sets won with point difference (P) & 1.209 & .069 & .555 & 17.522 & $\mathbf{. 0 1}$ \\
\hline Sets lost with point difference (P) & -.067 & .079 & -.028 & -.850 & .20 \\
\hline Games won played in five sets (P) & 1.569 & .141 & .345 & 11.117 & $\mathbf{. 0 1}$ \\
\hline Games lost played in five sets (P) & -.743 & .149 & -.150 & -4.968 & $\mathbf{. 0 1}$ \\
\hline Games won after 0-2 in sets for opponent (P) & .403 & .443 & .022 & .911 & .20 \\
\hline Games lost after leading 2-0 in sets (P) & -.064 & .449 & -.003 & -.142 & .20 \\
\hline
\end{tabular}

Legend: $R$ - Multiple correlation coefficient; $R^{2}$ - Determinantion coefficient; $R_{c}{ }^{2}$ - Corrected determination coefficient; $F$ - F-ratio; $p$ - Probability; B - Beta coefficient; $S E$ Standarda error of estimate; $\boldsymbol{B}$ - Beta standardize partial contribution; $\boldsymbol{t}$ - $\boldsymbol{t}$-test; D - Dependent; $\mathbf{P}$ - Predictor.

TABLE 6

Prediction of the variable sets won with the other game efficiency variables for the players in two SOKAZ table tennis championships in 2007.

\begin{tabular}{lccccc}
\hline \multirow{2}{*}{ Regression } & $\mathrm{R}$ & $\mathrm{R}^{2}$ & \multicolumn{1}{c}{$\mathrm{R}_{c}^{2}$} & \multicolumn{1}{c}{$F$} & \multicolumn{1}{c}{$p$} \\
\cline { 2 - 6 } & $\mathbf{. 7 8 6}$ & $\mathbf{. 6 1 8}$ & $\mathbf{. 6 1 6}$ & $\mathbf{2 5 6 . 2 9}$ & $\mathbf{. 0 1}$ \\
\hline Variables & \multicolumn{1}{c}{$\mathrm{B}$} & \multicolumn{1}{c}{$S E$} & \multicolumn{1}{c}{$\beta$} & \multicolumn{1}{c}{$t$} & \multicolumn{1}{c}{$p$} \\
\hline Sets won (D) & 19.531 & 2.770 & & 7.052 & $\mathbf{. 0 1}$ \\
\hline Sets won with point difference (P) & 3.725 & .204 & .535 & 18.228 & $\mathbf{. 0 1}$ \\
\hline Sets lost with point difference (P) & .404 & .234 & .053 & 1.728 & .05 \\
\hline Games won played in five sets (P) & 4.227 & .418 & .291 & 10.114 & $\mathbf{. 0 1}$ \\
\hline Games lost played in five sets (P) & -.245 & .443 & -.015 & -.553 & .20 \\
\hline Games won after 0-2 in sets for opponent (P) & 1.056 & 1.311 & .018 & .805 & .20 \\
\hline Games lost after leading 2-0 in sets (P) & -.372 & 1.331 & -.006 & -.279 & .20 \\
\hline
\end{tabular}

Legend: $R$ - Multiple correlation coefficient; $R^{2}$ - Determinantion coefficient; $R_{c}^{2}$ - Corrected determination coefficient; $F$ - F-ratio; $p$ - Probability; B - Beta coefficient; $S E$ Standarda error of estimate; $\boldsymbol{B}$ - Beta standardize partial contribution; $\boldsymbol{t}$ - $\boldsymbol{t}$-test; D - Dependent; $\mathbf{P}$ - Predictor. 
TABLE 7

Prediction of the variable game index with log variables

for the players in two SOKAZ table tennis championships in 2007.

\begin{tabular}{llllll}
\hline \multirow{2}{*}{ Regression } & $R$ & $R^{2}$ & $R_{c}^{2}$ & $F$ & \multicolumn{1}{c}{$p$} \\
\cline { 2 - 6 } & $\mathbf{. 6 7 0}$ & $\mathbf{. 4 4 8}$ & $\mathbf{. 4 4 7}$ & $\mathbf{2 5 2 . 8 2}$ & $\mathbf{. 0 1}$ \\
\hline Variables & $\mathrm{B}$ & $S E$ & $\beta$ & $t$ & \multicolumn{1}{c}{$p$} \\
\hline Game index (D) & -.036 & .021 & & -1.761 & .05 \\
\hline Turnover games (lost after leading 2-0 and won after 0-2) $(\mathrm{P})$ & .031 & .018 & .045 & 1.738 & .05 \\
\hline Games played in five sets (P) & .458 & .028 & .436 & 16.405 & $\mathbf{. 0 1}$ \\
\hline Sets played on point difference (P) & .558 & .037 & .394 & 15.866 & $\mathbf{. 0 1}$ \\
\hline
\end{tabular}

Legend: $R$ - Multiple correlation coefficient; $R^{2}$ - Determinantion coefficient; $R_{c}{ }^{2}$ - Corrected determination coefficient; $F$ - F-ratio; $p$ - Probability; B - Beta coefficient; $S E$ Standarda error of estimate; $\boldsymbol{B}$ - Beta standardize partial contribution; $\boldsymbol{t}$ - $\boldsymbol{t}$-test; D - Dependent; $\mathbf{P}$ - Predictor.

TABLE 8

Prediction of the variable games won with log variables

for the players in SOKAZ table tennis championships in 2007.

\begin{tabular}{lccccc}
\hline \multirow{2}{*}{ Regression } & $R$ & $R^{2}$ & $R_{c}^{2}$ & $F$ & $p$ \\
\cline { 2 - 7 } & $\mathbf{. 5 9 5}$ & $\mathbf{. 3 5 7}$ & $\mathbf{. 3 5 4}$ & $\mathbf{1 2 9 . 8 2}$ & $\mathbf{. 0 1}$ \\
\hline Variables & $\mathrm{B}$ & $S E$ & $\beta$ & $t$ & \multicolumn{1}{c}{$p$} \\
\hline Game won (D) & 6.040 & 1.289 & & 4.685 & $\mathbf{. 0 1}$ \\
\hline Turnover games (lost after leading 2-0 and won after 0-2) (P) & -.064 & .399 & -.006 & -.160 & .20 \\
\hline Games played in five sets (P) & .220 & .141 & .080 & 1.555 & .10 \\
\hline Sets played on point difference (P) & .703 & .063 & .536 & 11.103 & $\mathbf{. 0 1}$ \\
\hline
\end{tabular}

Legend: $R$ - Multiple correlation coefficient; $R^{2}$ - Determinantion coefficient; $R_{c}^{2}$ - Corrected determination coefficient; $F$ - F-ratio; $p$ - Probability; B - Beta coefficient; $S E$ Standarda error of estimate; $\boldsymbol{B}$ - Beta standardize partial contribution; $\boldsymbol{t}$ - $\boldsymbol{t}$-test; D - Dependent; $\mathbf{P}$ - Predictor.

TABLE 9

Prediction of the index total efficacy with log variables for the players in SOKAZ table tennis championships in 2007.

\begin{tabular}{llllll}
\hline \multirow{2}{*}{ Regression } & $\mathrm{R}$ & $\mathrm{R}^{2}$ & $\mathrm{R}_{c}^{2}$ & $F$ & \multicolumn{1}{l}{$p$} \\
\cline { 2 - 6 } & $\mathbf{. 1 5 6}$ & $\mathbf{. 0 2 4}$ & $\mathbf{. 0 2 0}$ & $\mathbf{5 . 8 4}$ & $\mathbf{. 0 1}$ \\
\hline Variables & $\mathrm{B}$ & \multicolumn{1}{c}{$S E$} & \multicolumn{1}{c}{$\beta$} & \multicolumn{1}{c}{$t$} & \multicolumn{1}{c}{$p$} \\
\hline Total efficacy $(\mathrm{D})$ & 1.290 & .057 & & 22.517 & $\mathbf{. 0 1}$ \\
\hline Turnover games (lost after leading 2-0 and won after 0-2) $(\mathrm{P})$ & -.018 & .018 & -.044 & -1.026 & .20 \\
\hline Games played in five sets (P) & .069 & .006 & .070 & 1.104 & .20 \\
\hline Sets played on point difference $(\mathrm{P})$ & .053 & .003 & .111 & 1.868 & .05 \\
\hline
\end{tabular}

Legend: $R$ - Multiple correlation coefficient; $R^{2}$ - Determinantion coefficient; $R_{c}{ }^{2}$ - Corrected determination coefficient; $F$ - F-ratio; $p$ - Probability; B - Beta coefficient; $S E$ Standarda error of estimate; $\boldsymbol{B}$ - Beta standardize partial contribution; $\boldsymbol{t}$ - $\boldsymbol{t}$-test; D - Dependent; $\mathbf{P}$ - Predictor. 


\section{DISCUSSION}

The main research findings reached the conclusion that, even with a small number of indexes and variables derived directly from the results of table tennis games, a statistically significant correlation with the final result in table tennis games (winning a game or set), can be found. Moreover, compared with previous research provided by Sindik and Kondrič (2011), the results are (with minor exceptions) almost identical to the results conducted one year ago on an almost identical sample. On the one hand, these results confirm the results of the previous research, thus indicating the »stability of the predictive characteristics of the index and variables deduced directly from the results of table tennis games in the league championships and for the so-called direct efficacy indicators. On the other hand, the results are probably also stable characteristics of the score achievement of table tennis players in a large-scale table tennis competition, such as the one in SOKAZ (Association of Recreational Table Tennis Players of Zagreb), as same for the elite table tennis competitions.

With regards to the inter-correlation of efficiency indexes and variables on the basis of competition results (Table 2), many statistically significant correlations of »direct « indicators of efficiency are noticeable. Thus, direct indicators of efficiency (game index, set index, games won, games lost, sets won, and sets lost) are medium or strongly and significantly correlated with other indexes and variables. It is interesting that the variables indicating the number of defeats (in sets or matches) are also statistically correlated significantly and positively (although with somewhat lower values) with direct efficacy indexes. However, it was evident that the turnover index is the least correlated with direct efficacy indicators (correlations are statistically significant, but low) of all the indirect indicators of competitive efficacy (turnover index and associated variables, sets won with point difference and associated variables, games won played in five sets index, and associated variables). Even though the correlation is spurious, it was clear that the turnover index is highly and positively correlated with the total efficacy index, which is information providing a guideline for future research. The total efficacy index is statistically and positively correlated with all efficacy indexes, as well as with variables indicating games and sets won: sets won with point difference, games won played in five sets, and games won after 0-2 in sets for opponent. However, correlations of the total efficacy index are negative or zero in relation to variables indicating games and sets lost: sets lost with point difference, games lost played in five sets, and games lost after leading 2-0 in sets; therefore also to variables that are indirect efficacy indexes, and especially to the indexes deriving from them. An interesting point is that all direct and indirect efficacy indexes are negatively (although weakly, but significantly) correlated with the league level; therefore, even in the "weaker" competitive leagues these indexes have lower values. This can indirectly imply information about varying levels of uncertainty for competitions in »stronger" or »weaker« leagues (Sindik \& Vidak, 2009).

Observing Tables 3 to 6 it is clear that all direct efficacy indicators of table tennis players (variables and indexes) can be successfully predicted based on indirect efficacy indicators. The most successful predictors are sets won with point difference (variable) and the sets won with point difference index, while the least successful predictors (at the same time also statistically insignificant) are the turnover index and games won after 0-2 in sets for opponent, or rather games lost after leading 2-0 in sets. This information can probably be explained by the fact that big result turnovers are a fairly rare event in table tennis games. On the other hand, sets won with point difference are events occurring somewhat more frequently than games won played in five sets.

For Tables 7 and 8, one can deduce that, among predictors that are neutral or log variables, the total number of sets played on point difference is the best predictor of direct indicators of efficiency: games won and game index. Therefore, the total number of sets played on point difference is the best predictor of result success, which is also reflected in successful prediction of the total efficacy index (Table 9). (For the total efficacy index as a criterion, we did not show predictions based on the indirect efficacy indexes of table tennis players because predictions based on all indirect indexes of a table tennis player's success are spurious, even though they registered as statistically significant. That is to say, the total efficacy index is obtained by a simple linear combination of three indirect efficacy indexes.)

One relevant fault of all efficiency indicators (indexes and variables) directly derived from competition results is that the total result does not necessarily need to be a real "measure" of player competitive efficiency. In practical situations in competition, players might be "laid-back" in situations of more significant result advantage or might "hold back" in relation to an opponent, or there could be "predictions" of convincing victory or defeat, which result in "playing" with anticipated inferior or "superior" opponents during the entire event (Sindik \& Juričević, 2007). We should not forget that we are not talking about top-quality table tennis, but recreational table tennis (our sample of subjects); therefore we should be additionally careful in generalizing these results. 
However, on average and in competitions that are more equal in terms of results, the suggested efficiency indicators could be useful.

On the basis of these results, one could cautiously presume that winning in sets won with point difference and associated variables and index, as well as winning in games played in five sets are »more critical« for the final outcome of the game (games won, sets won, game index, total efficacy index), at least in the case of this sample. It is not only wins in sets played on point difference, but also the number of sets played on point difference itself (as a log variable), that can predict in a statistically significant manner the direct efficacy indexes of table tennis players, and also the total efficacy index. Thus, we can consider that sets played on point difference (just as games played in five sets, and, with a lower impact - turnover games) could be very important elements in understanding the efficacy of the table tennis player in a sequence of games. On the basis of analysis of such elements, it would be necessary (for a specific sample of table tennis players) to prepare two more basic strategies when planning a training transformation process (possibly "psychologically" decisive for the final outcome of an individual table tennis game).

First, to attempt to steer players towards focusing on the importance of maximum optimal motor performance and psychological engagement in the decisive part of an individual table tennis game: with the goal of winning the set played on point difference (at the level of a sequence of a few games, focus is needed on potential games played in five sets and potential turnover games). In other words, an individual has to be focused to win in "sensitive" game situations (set played on point difference), and must avoid "easing off" when leads in points and sets occur (games played in five sets and turnover games). Second, to assure the optimal motor performance and psychological stability of an individual in situations when an unfavourable outcome has already occurred (when a player has lost a game played in five sets or a turnover game, or when a player has lost a set played in point difference).

This study examined different players at varying levels of competitive quality (in relation to the competition effect) and different levels of quality in their opponents, which is a significant improvement in relation to earlier research studies. Moreover, we tested a much larger sample, practically the whole population of table tennis players in the recreational leagues of SOKAZ (who have played a minimum of 8 games per year).

However, it is desirable in any case to inspect the proposed variables and indexes (direct and indirect indicators) of player efficacy in future research, on a sample of elite table tennis players, perhaps not only male, and also from different age groups. We could also use more indirect indicators of player efficacy, as did Sindik and Juričević (2007), on the two sets won and 21 points won point-system.

\section{CONCLUSION}

As a conclusion, it was demonstrated that correlations between individual variables of a table tennis competitor's efficiency are statistically significant (1), as well as the correlations between indexes of a table tennis competitor's efficiency are also statistically significant and positive (2). The total efficiency index was significantly high and moderately positively associated with all major variables that are indicators ofthe efficiency of table tennis players: won games, games won after 0-2 in sets for the opponent, sets won with the difference, and games won played in five sets (3). In particular, we found positive (and significant) correlations between all the direct and indirect indicators of player efficacy (medium high to high correlated). Finally, indexes and variables directly derived from the results can predict indexes and variables of final competition success to a statistically significant degree: game index, set index, games won, and sets won. Log variables can predict the total index of final competitive success to a statistically significant degree: total efficacy index.

\section{REFERENCES}

Baca, A., Baron, R., Leser, R., \& Kain, H. (2004). A Baca, A., Baron, R., Leser, R., \& Kain, H. (2004). A process oriented approach for match analysis in table tennis. In A. Lees, J. F. Kahn, and I. Maynard (Eds.), Science and Racket Sports III (pp. 214-219). London and New York: Routledge.

Brčić, B., Viskić Štalec, N., \& Jaklinović Fressl, Ž. (1997). Predictive value of variables for the evaluation of technical-tactical elements in hand ball. Kinesiology, 1(29), 60-70.

Đokić, Z. (2007). IT'TF scored a goal (changes in rules of table tennis during 2000-2003). In M. Kondrič and G. Furjan-Mandić (Eds.), Proceedings book of the 10th Anniversary International Table Tennis Sports Science Congress, Zagreb, 18.-20.5.2007 (pp. 168-174). Zagreb, HR: University of Zagreb - Faculty of Kinesiology, Croatian Table Tennis Association, International Table Tennis Association.

Franks, I. M., \& Miller, G. (1991). Training coaches to observe and remember. Journal of Sports Science, 9(3), 285-297. doi: 10.1080/026404 19108729890; PMid:1960799 
Grujić, A. (1975). Stoni tenis [Table tennis]. Beograd, SRB: Savez za fizičku kulturu Jugoslavije.

Hudetz, R. (1984). Stoni tenis - tebnika [Table tennistechniques]. Zagreb, CRO: Sportski forum.

Hughes, M. D., \& Franks, I. M. (1997). Notational Analysis of Sport. London, GBR: E. \& F. N. Spon.

Leser, R., \& Baca, A. (2009). Practice oriented match analysis in table tennis as coaching aid. In A. Lees, D. Cabello and G. Torres (Eds.), Science and Racket Sports IV (pp. 214-219). London and New York: Routledge.

Sindik, J. (1999). Zavisnost rezultata u stolnom tenisu od nekih pokazatelja uspješnosti igre [Correlation of results in table tennis and some indicators of playing efficiency]. In D. Milanović (Ed.), Proceedings of the 2nd international conference "Kinesiology - Science for the 21st century", Dubrounik (pp. 302-305). Zagreb, CRO: Faculty of Physical Education, University of Zagreb.

Sindik, J., \& Juričević, M. (2007). Dependence of results in table tennis on certain game efficiency indexes. In M. Kondrič and G. Furjan-Mandić (Eds.), Proceedings book of the 10th Anniversary International Table Tennis Sports Science Congress, Zagreb, 18.-20.5.2007 (pp. 427-435). Zagreb, HR: University of Zagreb - Faculty of Kinesiology,
Croatian Table Tennis Association, International Table Tennis Association.

Sindik, J., \& Kondrič, M. (2011). Correlation between the result efficiency indexes and success in table tennis. International Journal of Performance Analysis in Sport, 11(2), 268-284.

Sindik, J., \& Vidak, N. (2009). Uncertainty coefficient as a method for optimization of the competition system in table-tennis leagues in „SOKAZ“. Sport Science, 2(2), 66-72.

Wilson, K. \& Barnes, C. A., (1998). Reliability and validity of a computer based notational analysis system for competitive table tennis. In A. Lees, I. Maynard, M. Hughes, and T. Reilly (Eds.), Science and Racket Sports II (pp. 263-268). London and New York: Routledge.

Wu Xiao, Z., \& Escobar-Vargas, J. (2007).

Notational analysis for competition in table tennis (part 1): Based format analysis. In M. Kondrič and G. Furjan-Mandić (Eds.), Proceedings book of the 10th Anniversary International Table Tennis Sports Science Congress, Zagreb, 18.20.5.2007 (pp. 104-108). Zagreb, CRO: University of Zagreb - Faculty of Kinesiology, Croatian Table Tennis Association, International Table Tennis Association.

Received: November 11, 2011 Revision received: April 15, 2012

Accepted: April 30, 2012

Correspodence to: Joško Sindik, PhD

Institute for Anthropological Research

Ljudevita Gaja 32 10000 Zagreb, Croatia

E-mail: josko.sindik@inantro.hr Phone: 0038615207737 Fax: 0038615207740 